\title{
Evolution of Conjectures in Cournot Oligopoly*
}

\author{
Jacco Thijssen ${ }^{\dagger}$
}

This version, November 2003.

First version, March 2002

\begin{abstract}
This paper considers a dynamic market where a fixed number of firms engages in Cournot oligopoly. Firms choose the output level based on their assessment of the competitors' reaction to their output choice. This is parameterized using an approach reminiscent of conjectural variations. On a second level firms adapt their conjectural variation by imitating the most successful firm. Simulations suggest that in the long-run the Walrasian, Cournot-Nash and cartel equilibria survive. The theory of nearly-complete decomposability is used to show that the Walrasian equilibrium is approximately the only stochastically stable state.
\end{abstract}

Keywords: Oligopoly theory, conjectural variations, stochastic stability, decomposability

JEL classification: L13, C73

*I thank Dolf Talman for his meticulous proof reading and inspiring discussions. Helpful comments and suggestions from Peter Kort and Marieke Quant are greatly appreciated. The usual disclaimer applies.

${ }^{\dagger}$ Department of Economics, Trinity College Dublin, Dublin 2, Ireland, Jacco.Thijssen@tcd.ie. 


\section{Introduction}

The question under which conditions collusion or cartel formation in oligopolistic markets is possible is an important issue in the theory of industrial organisation. It is particularly interesting for anti-trust policies. There are several ways in which the issue has been addressed in the literature. The static Cournot or Bertrand models don't allow for a cooperative outcome. Experimental evidence, e.g. by Friedman (1967), Axelrod (1984), and Offerman et al. (2002), suggests that cooperation or cartel formation (tacit collusion) does arise in oligopolistic markets.

In the literature many contributions try to explain sustainability of tacit collusion. For most of these models it holds that extensions to the basic static model are needed in order to get cooperation as a possible equilibrium outcome, e.g. by assuming incomplete information or asymmetries in the firms' technologies. There is, however, a very simple way of obtaining a collusive outcome in the static model, namely by introducing conjectural variations, a concept that dates back to Bowley (1924). This approach assumes that firms take into account the reaction of the market to their own quantity choice. For example, the standard best-reply dynamics is compatible with conjectural variations stating that the market does not respond to one's own actions. The problem is that conjectural variations are essentially a dynamic concept, but are mostly used in a static environment. Kalai and Stanford (1985) show that there are repeated game formulations of Cournot markets that can result in beliefs in the spirit of conjectural variations without abandoning full rationality. In a recent paper Friedman and Mezzetti (2002) show how conjectural variations can be used in an oligopoly model. They consider a differentiated product market with boundedly-rational, price-setting firms that at each point in time maximise their profit over an infinite time horizon based on a conjectural variation. These authors also study an adaptation process of the conjectural variation where adaptation takes place if the observed price change is substantially larger than the price change predicted by the conjectural variation. They show that under certain conditions the Nash equilibrium always constitutes a stable steady-state. Furthermore, as substitutability among firms increases, a more cooperative outcome can be sustained as a stable steady-state. In the limit (perfect substitutability) this leads to full cooperation.

An important step in the evolution of economic thinking has been the interest of economists for the theory of evolution applied to economic phenomena. Several contributions to the literature have set off the development of this field. First there is the concept of bounded rationality introduced by Herbert Simon (cf. Simon (1957)). The bounded rationality approach replaces the assumption of full rationality of 
economic agents. Secondly, in neo-classical economics the objective of agents is to maximise some absolute quantity (e.g. utility or profit), whereas Alchian (1950) already pointed out that relative payoffs are often of more interest.

In this paper, the concept of stochastic stability introduced by Foster and Young (1990) is used to analyse an oligopoly model with boundedly rational firms which are ultimately interested in relative profits. The first paper in industrial organisation which applies the concept of stochastic stability is Vega-Redondo (1997). He considers a Cournot oligopolistic market where firms choose their quantity level by imitating the most successful firm, i.e. the firm that makes the highest profit. He shows that the unique stochastically stable state is given by the Walrasian equilibrium. This model has been extended by Alós-Ferrer et al. (1999) by allowing for entry and exit. They find that if there are decreasing returns to scale, then the market will eventually settle in a Walrasian equilibrium. In case of increasing returns to scale, a monopoly will arise eventually.

As mentioned before, an experimental study by Offerman et al. (2002) shows that not only the Walrasian equilibrium may survive in a Vega-Redondo framework, but also the collusive (cartel) equilibrium. This experimental evidence seems to suggest that the behavioural assumptions in Vega-Redondo (1997) are too restrictive. There are attempts in the literature to construct models with more behavioural rules. For example, Schipper (2003) models myopic optimisers (best-repliers) and profit imitators à la Vega-Redondo (1997). He finds that the market eventually converges to a situation where the myopic optimisers play a best-reply to the imitators and the imitators play a semi-Walrasian quantity taking into account the existence of the best-repliers. In this model, neither the Walrasian equilibrium nor the cartel outcome is obtained. Kaarbøe and Tieman (1999) use a similar model to show that in supermodular games a Nash equilibrium is always selected in the limit.

In all the papers mentioned above agents either cannot change their behaviour, or changing behaviour is modelled as an exogenous random process. The model presented in this chapter gives more flexibility to the behavioural assumptions underlying the results of these papers by endogenising the behavioural choice. It is assumed that firms base their quantity choice in a boundedly rational way on observations from the past and on their conjectures about competitors' reactions to their behaviour. The latter aspect is modelled by using a variable that measures the supposed - immediate reaction of others to one's own actions. The quantity dynamics is then modelled in such a way that a firm chooses the quantity that maximises next period's profit given the quantity choices of the previous period and its own conjecture. Hence, at the quantity level, firms are assumed to be myopic optimisers. This quantity dynamics is extended with random noise to capture aspects of the 
quantity choice that are not explained by the model. One can think for example of experimentation by firms which leads to a different quantity choice than would be expected from myopic optimisation. The noise part can also be interpreted as firms making mistakes in their myopic optimisation process. It might also capture the fact that a firm is replaced by a new firm that has the same conjecture, but starts with another quantity choice. The last aspect reflects what biologists call mutation.

It may happen that after some time a firm realises that it is making less profit than its competitors. In such a case, its conjecture is apparently not correct. In such cases, firms adapt their conjecture. They make this decision in a boundedly rational way, namely by imitating the conjecture of the firm that has the highest profit. This dynamics is called the conjecture dynamics. Here too a random noise term is added to capture experiments, mistakes or mutations. By allowing evolution at the behavioural level this paper is related to the literature on indirect evolution (cf. Güth and Yaari (1992)). The main difference is that in this paper it is not assumed that given a behavioural pattern agents act rationally. Boundedly rational behaviour enters here at both the behavioural and the quantity-setting level.

The quantity dynamics and the conjecture dynamics, together with the noise terms, lead to an ergodic Markov chain having a unique invariant probability measure. A simulation study is conducted to study the behaviour of this Markov chain. It is found that the cartel, Cournot-Nash, and Walrasian equilibria are played more frequently than other quantities. Furthermore, in the long-run the Walrasian equilibrium gets played more often.

To see if the Walrasian equilibrium can theoretically be supported as the most likely long-run outcome of the model, we look at the stochastically stable states of the Markov chain. The stochastically stable states are the states in the support of the unique invariant probability measure of the limit Markov chain when the error probabilities for both the quantity dynamics and the conjecture dynamics converge to zero. Due to the complexity of the dynamics it is not possible to get analytical results on the stochastically stable states. However, by construction of the model the Markov chain can be decomposed in a chain that governs the quantity dynamics and a chain that describes the conjecture dynamics. It is shown that - given the conjectures - the quantity dynamics has a unique invariant probability measure. The Markov chain is then aggregated over the quantity level using this measure and a Markov chain is obtained that is solely based on the conjecture dynamics. It is shown that this aggregated Markov chain has a unique invariant probability measure. The theory of nearly-complete decomposability as developed by Simon and Ando (1961), Ando and Fisher (1963) and Courtois (1977) provides conditions under which the invariant measure of the aggregated chain is an approximation of 
the invariant measure of the original chain.

Following this path of analysis it is obtained that the market will eventually settle in the Walrasian equilibrium, just like in Vega-Redondo (1997). So, even with the more elaborate behavioural structure of this model, the Walrasian equilibrium is still, by approximation, the only stochastically stable state. This results from the fact that imitative behaviour drives cooperative behaviour out, just as in Vega-Redondo (1997) profit imitation drives out cooperative quantity setting. This result only holds, however, if the frequency of the conjecture dynamics is sufficiently low. Then, the quantity dynamics has time to settle in its equilibrium. Otherwise, anything can happen, although the simulations suggest that the dynamics mainly evolves around the cartel, Cournot-Nash and Walrasian equilibria.

The chapter is organised as follows. In Section 2 the model is formally introduced. Section 3 presents a simulation study, whereas in Section 4 the model is analysed analytically. Section 5 concludes.

\section{The Model}

Let be given a dynamic market for a homogeneous good with $n$ firms, indexed by $I_{n}=\{1,2, \ldots, n\}$. At each point in time, $t \in \mathbb{N}$, competition takes place in a Cournot fashion, i.e. by means of quantity setting. Inverse demand is given by a smooth function $P: \mathbb{R}_{+} \rightarrow \mathbb{R}_{+}$satisfying $P^{\prime}(\cdot)<0$. The production technology is assumed to be the same for each firm and is reflected by a smooth cost function $C: \mathbb{R}_{+} \rightarrow \mathbb{R}_{+}$, satisfying $C^{\prime}(\cdot)>0$. If at time $t \in \mathbb{N}$ the vector of quantities is given by $q \in \mathbb{R}_{+}^{n}$, the profit for firm $i \in I_{n}$ at time $t$ is given by

$$
\pi\left(q_{i}, q_{-i}\right)=P\left(q_{i}+Q_{-i}\right) q_{i}-C\left(q_{i}\right)
$$

where $q_{-i}=\left(q_{j}\right)_{j \neq i}$ and $Q_{-i}=\sum_{j \neq i} q_{j}$.

Each firm $i \in I_{n}$ chooses quantities from a finite grid $\Gamma_{i}$. Define $\Gamma=\prod_{i \in I_{n}} \Gamma_{i}$. For further reference let $q(k), k=1, \ldots, m$, be the $k$-th permutation of $\Gamma$. It is assumed that in setting their quantities firms conjecture that their change in quantity results in an immediate change in the total quantity provided by their competitors. This can also be seen to reflect the firm's conjecture of the competitiveness of the market. Formally, firm $i \in I_{n}$ conjectures a value for the partial derivative of $Q_{-i}$ with respect to $q_{i}$. Using this conjecture, the firm wants to maximise next period's profit. Hence, the firm is a myopic optimiser, which reflects its bounded rationality. The first-order condition for profit maximisation of firm $i$ reads

$$
P^{\prime}\left(q_{i}+Q_{-i}\right)\left(1+\frac{\partial Q_{-i}}{\partial q_{i}}\right) q_{i}+P\left(q_{i}+Q_{-i}\right)-C^{\prime}\left(q_{i}\right)=0 .
$$


As can be seen from eq. (1) we assume that there is only a first order conjecture effect. Furthermore, we assume that it is linear. These assumptions add to the firm's bounded rationality. ${ }^{1}$

To facilitate further analysis, the conjectures are parameterised by a vector $\alpha \in$ $\mathbb{R}^{n}$ such that for all $i \in I_{n}$

$$
\left(1+\alpha_{i}\right) \frac{n}{2}=1+\frac{\partial Q_{-i}}{\partial q_{i}}
$$

Given a vector of conjectures an equilibrium for the market is given by $q \in \mathbb{R}_{+}^{n}$ such that for all $i \in I_{n}$ the first-order condition (1) is satisfied. Note that if all firms $i \in I_{n}$ have a conjecture $\alpha_{i}=-1$, the equilibrium coincides with the Walrasian equilibrium. Furthermore, if all firms have $\alpha_{i}=\frac{2-n}{n}$ or $\alpha_{i}=1$, the equilibrium coincides with the Cournot-Nash equilibrium or the cartel equilibrium, respectively. Therefore, the conjectures $\alpha_{i}=-1, \alpha_{i}=\frac{2-n}{n}$, and $\alpha_{i}=1$ will be called the Walrasian, Cournot-Nash, and cartel conjectures, respectively.

Each firm chooses its conjecture from a finite grid $\Lambda$ on $[-1,1]$, where it is assumed that $\Lambda \supset\left\{-1, \frac{2-n}{n}, 1\right\}$. The bounds of this finite grid represent the extreme cases of full competition $(\alpha=-1)$ and cartel $(\alpha=1)$. For further reference, let $\alpha(I), I=1, \ldots, N$, be the $I$-th permutation of $\Lambda^{n}=\prod_{i \in I_{n}} \Lambda$.

The dynamics of the market takes place in discrete time and consists of both a quantity dynamics and a conjecture dynamics. The quantity dynamics works as follows. At the beginning of period $t \in \mathbb{N}$, each firm gets the opportunity to revise its output with probability $0<p<1$. The output is chosen so that it maximises this period's profit based on last period's quantities and the firm's conjecture. That is, firm $i \in I_{n}$ seeks to find $q_{i}^{t} \in \Gamma_{i}$ so as to approximate as closely as possible the first-order condition from eq. (1), i.e. $q_{i}^{t} \in B\left(q_{-i}^{t-1}, \alpha_{i}^{t-1}\right)$, where for $q_{-i} \in \prod_{j \neq i} \Gamma_{j}$ and $\alpha_{i} \in \Lambda_{i}$,

$$
B\left(q_{-i}, \alpha_{i}\right)=\underset{q \in \Gamma_{i}}{\arg \min }\left\{\left|P^{\prime}\left(q+Q_{-i}\right)\left(1+\alpha_{i}\right) \frac{n}{2} q+P\left(q+Q_{-i}\right)-C^{\prime}(q)\right|\right\} .
$$

If there are ties, firm $i$ chooses any quantity from $B\left(q_{-i}^{t-1}, \alpha_{i}^{t-1}\right)$ using a probability measure $\eta_{i}(\cdot)$ with full support. The dynamics described above constitutes the pure quantity dynamics. The actual quantity choice can be influenced by several other aspects. For example, a firm can experiment and choose another quantity. Another possibility is that firms make mistakes in their optimisation process. Finally, a firm may be replaced by a new firm that has the same conjecture, but sets a different

\footnotetext{
${ }^{1}$ The first-order and linearity assumptions are also made throughout the static literature on conjectural variations. This seems incompatible with the assumption of fully rational firms in these models.
} 
quantity to start with. This is what biologists refer to as mutation. To capture these effects we assume that each firm experiments or makes a mistake with probability $\varepsilon>0$ and chooses any quantity from $\Gamma_{i}$ using a probability measure $\nu_{i}(\cdot)$ with full support. Given a conjecture vector $\alpha(I)$ for some $I \in\{1, \ldots, N\}$ and an error probability $\varepsilon$, the quantity dynamics gives rise to a Markov chain on $\Gamma$ with transition matrix $M_{I}^{\varepsilon}$, a typical element of which is given by

$$
\begin{aligned}
M_{I}^{\varepsilon}(k, l)=\prod_{i \in I_{n}} & \left\{( 1 - \varepsilon ) \left[p \mathbb{1}_{\left(q_{i}(l) \in B\left(q_{-i}(k), \alpha_{i}(I)\right)\right)} \eta_{i}\left(q_{i}(l)\right)\right.\right. \\
+ & \left.\left.(1-p) \mathbb{1}_{\left(q_{i}(k)=q_{i}(l)\right)}\right]+\varepsilon \nu_{i}\left(q_{i}(l)\right)\right\},
\end{aligned}
$$

where $\mathbb{1}_{(\cdot)}$ denotes the indicator function and the part between square brackets gives the transition probabilities for the pure quantity dynamics.

The conjecture dynamics takes place at the end of period $t$, when each firm $i$ gets the opportunity to revise its conjecture with probability $0<\tilde{p}<1$. The idea behind this revision is that once in a while a firm analyses its past performance and it assesses the correctness of its conjecture by looking at the performance of the other firms. It is assumed that each firm can observe the individual quantity choices of its competitors and therefore it can also deduce the conjectures that its competitors use. It can then imitate the firm that made the highest profit in period $t$. Since deriving the conjectures requires effort we assume that firms change their conjecture less often than their quantity choice which is reflected in assuming that $\tilde{p}<p .^{2}$ Formally, firm $i$ 's choice $\alpha_{i}^{t}$ is such that $\alpha_{i}^{t} \in \tilde{B}\left(\alpha^{t-1}, q^{t}\right)$, where for given $\alpha \in \Lambda^{n}$ and $q \in \Gamma$,

$$
\tilde{B}(\alpha, q)=\underset{\gamma \in \Lambda}{\arg \max }\left\{\exists_{j \in I_{n}}: \alpha_{j}=\gamma, \forall_{k \in I_{n}}: \pi\left(q_{j}, q_{-j}\right) \geq \pi\left(q_{k}, q_{-k}\right)\right\} .
$$

If there are ties, firm $i$ chooses any element from $\tilde{B}\left(\alpha^{t-1}, q^{t}\right)$ using a probability measure $\tilde{\eta}_{i}(\cdot)$ with full support. This dynamic process is called the pure conjecture dynamics. As in the quantity dynamics we allow for mistakes or experimentation. So, each firm chooses with probability $\tilde{\varepsilon}>0$ any conjecture using a probability measure $\tilde{\nu}_{i}(\cdot)$ with full support. For each $k \in\{1, \ldots, m\}$ and corresponding quantity vector $q(k)$, and error probability $\tilde{\varepsilon}$, the conjecture dynamics gives rise to a Markov chain on $\Lambda^{n}$ with transition matrix $\lambda_{k}^{\tilde{\varepsilon}}$. The transition probability from $\alpha(I)$ to $\alpha(J)$

\footnotetext{
${ }^{2}$ One could argue that since the firm can derive its competitors' conjectures it can always optimally react. However, it would have to gather more information than just quantity choices. This requires effort and, hence, costs. Therefore, we assume that conjecture updating takes place less often than the application of the rule of thumb of quantity adjustment with fixed conjectures.
} 
is given by

$$
\begin{aligned}
\lambda_{k}^{\tilde{\varepsilon}}(I, J)=\prod_{i \in I_{n}} & \left\{( 1 - \tilde { \varepsilon } ) \left[\tilde{p} \mathbb{1}_{\left(\alpha_{i}(J) \in \tilde{B}(\alpha(I), q(k))\right)} \tilde{\eta}_{i}\left(\alpha_{i}(J)\right)\right.\right. \\
& \left.\left.+(1-\tilde{p}) \mathbb{1}_{\left(\alpha_{i}(J)=\alpha_{i}(I)\right)}\right]+\tilde{\varepsilon} \tilde{\nu}_{i}\left(\alpha_{i}(J)\right)\right\},
\end{aligned}
$$

where the part between square brackets gives the transition probabilities for the pure conjecture dynamics.

The combined quantity and conjecture dynamics yield a Markov chain on $\Gamma \times \Lambda^{n}$ with transition matrix $Q^{\varepsilon, \tilde{\varepsilon}}$. Entries in this transition matrix are grouped according to the conjecture index. So, the $k$-th row in $Q^{\varepsilon, \tilde{\varepsilon}}$ consists of the transition probabilities from the state with conjectures $\alpha(1)$ and quantities $q(k)$. The $m *(I-1)+k$-th row contains the transition probabilities from the state with conjectures $\alpha(I)$ and quantities $q(k)$. A typical element of $Q^{\varepsilon, \tilde{\varepsilon}}$ is given by

$$
Q^{\varepsilon, \tilde{\varepsilon}}\left(k_{I}, l_{J}\right)=M_{I}^{\varepsilon}(k, l) \lambda_{k}^{\tilde{\varepsilon}}(I, J)
$$

which should be read as the transition probability form the state with conjectures $\alpha(I)$ and quantities $q(k)$ to the state with conjectures $\alpha(J)$ and quantities $q(l)$.

\section{A Simulation Study}

Since for all $i \in I_{n}$, the probability distributions $\nu_{i}(\cdot)$ and $\tilde{\nu}_{i}(\cdot)$ have full support, the Markov chain $Q^{\varepsilon, \tilde{\varepsilon}}$ is ergodic and, hence, has a unique invariant probability measure. To gain some insight in the long-run behaviour of the Markov chain a simulation study has been conducted. The demand side of the market is described by an inverse demand function given by

$$
P(Q)=45-\sqrt{3 Q} .
$$

It is assumed that all firms have access to the same technology which is represented by the cost function

$$
C(q)=q \sqrt{q} .
$$

This description is the same as the one used in the experiment conducted by Offerman et al. (2002). The focus in that paper is on the frequencies of the Walrasian (full competition), Cournot-Nash, and cartel quantities, respectively. The Walrasian quantity, denoted by $q^{w}$, solves the inequality

$$
P\left(n q^{w}\right) q^{w}-C\left(q^{w}\right) \geq P\left(n q^{w}\right) q-C(q) \quad \forall q \in \mathbb{R}_{+} .
$$

The Cournot-Nash $\left(q^{n}\right)$ and cartel $\left(q^{c}\right)$ quantities solve

$$
P\left(n q^{n}\right) q^{n}-C\left(q^{n}\right) \geq P\left((n-1) q^{n}+q\right) q-C(q) \quad \forall q \in \mathbb{R}_{+},
$$


and

$$
P\left(n q^{c}\right) q^{c}-C\left(q^{c}\right) \geq P(n q) q-C(q) \quad \forall q \in \mathbb{R}_{+},
$$

respectively. Following Offerman et al. (2002) and taking $n=3$ firms, these quantities are given by $q^{w}=100, q^{n}=81$, and $q^{c}=56.25$, respectively. The Walrasian, Cournot-Nash, and cartel conjectures equal $-1,-1 / 3$, and 1 , respectively.

For the simulation, the quantity grid for all firms is taken to be $\Gamma=\{49,50, \ldots, 108\}$ and the quantity grid is set to $\Lambda=\left\{-1,-\frac{1}{3}, 1\right\}$. This implies that the state space is $5,832,000$ dimensional. The probabilities of quantity and conjecture adaptation are set to $p=0.9$ and $\tilde{p}=0.4$, respectively. We simulate 200 runs of 150 time periods. For each simulation the error probabilities $\varepsilon$ and $\tilde{\varepsilon}$ are iid uniformly drawn from the interval $[0.001,0.2]$.

The frequencies of the conjectures and the running frequencies of the aggregate quantities are shown in Figure 1. The running frequency for quantity $Q$ has window size four, i.e. it is the frequency of all observations in the set $\{Q-4, Q-3, \ldots, Q+4\}$. As can be seen from this figure, the Walrasian quantity has the highest frequency,
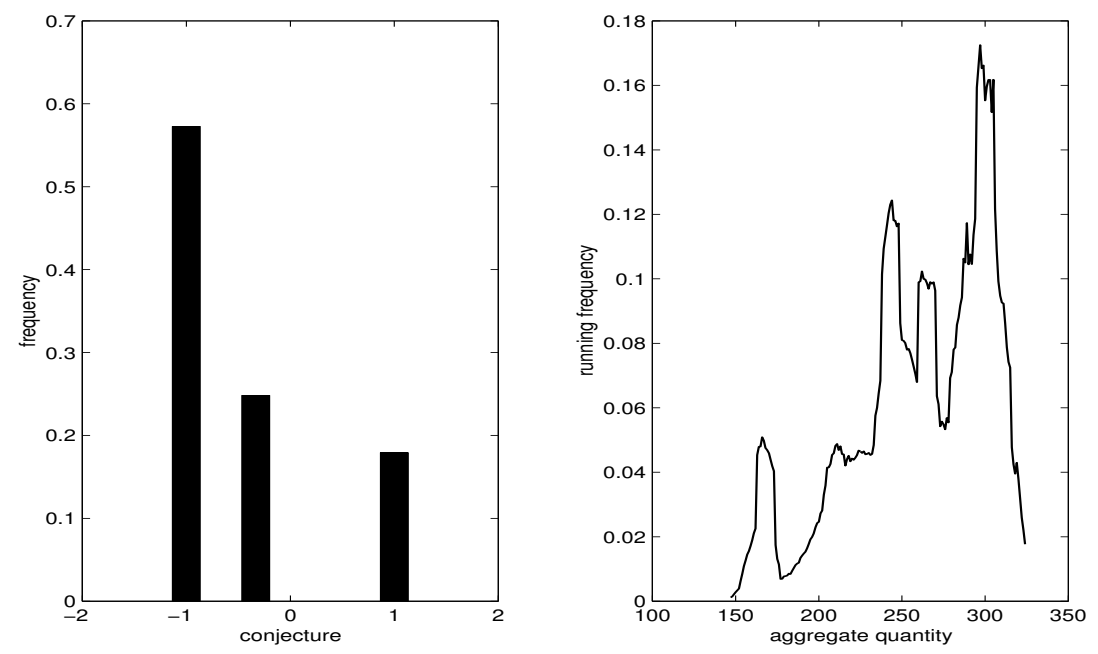

Figure 1: Frequencies of conjectures (left panel) and running frequencies of aggregate quantities (right panel).

with two other peaks at the Cournot-Nash quantity and the cartel quantity. The frequencies of the conjectures chosen paint a similar picture.

If the sample is split into short-run (first 50 periods of each run) and long-run (last 50 periods of each run) data, the frequencies are as depicted in Figures 2 and 3. These findings show that all three equilibria are being played in the long-run. If we look at prices, one of the simulation runs led to a price-run depicted in Figure 4. This price-run stays very close to Offerman et al. (2002) who report that both the 

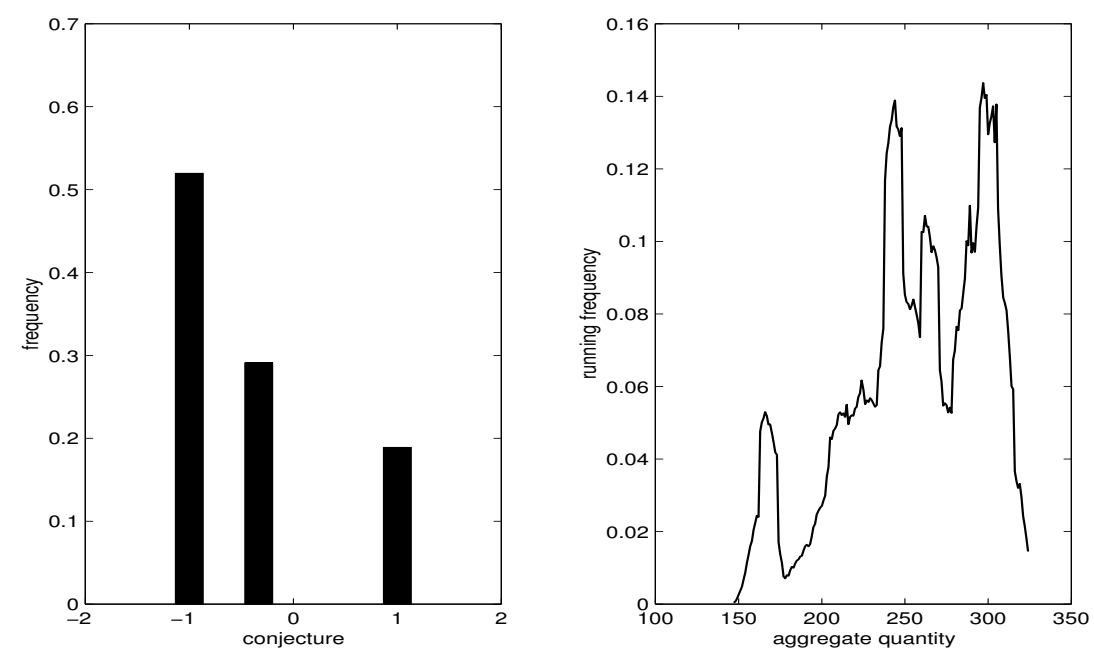

Figure 2: Short-run frequencies of conjectures (left panel) and running frequencies of aggregate quantities (right panel).
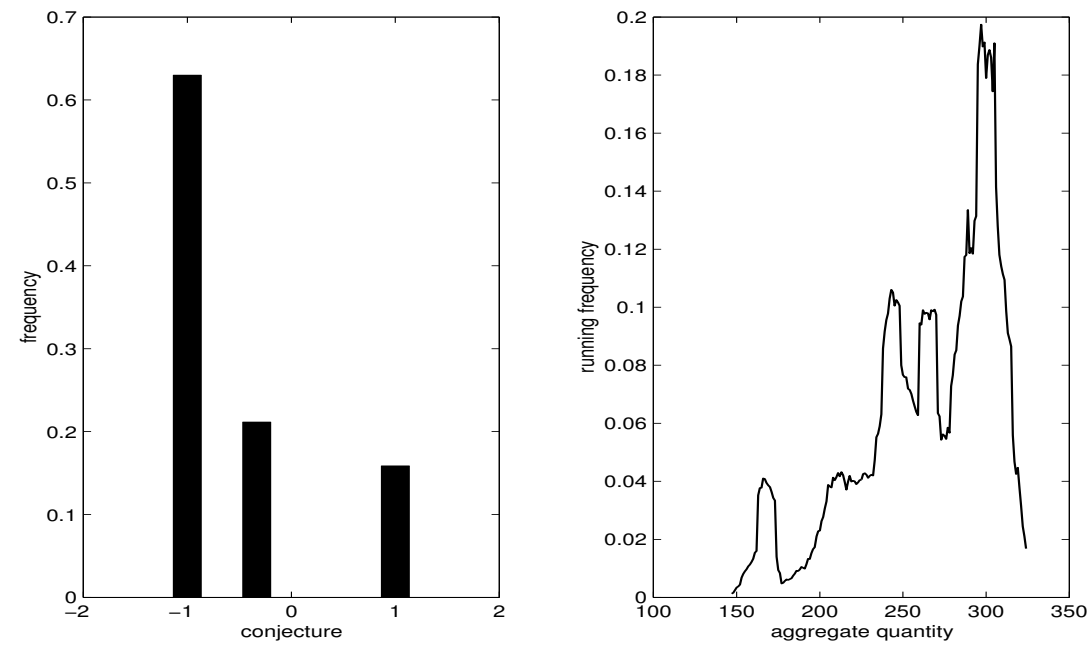

Figure 3: Long-run frequencies of conjectures (left panel) and running frequencies of aggregate quantities (right panel). 


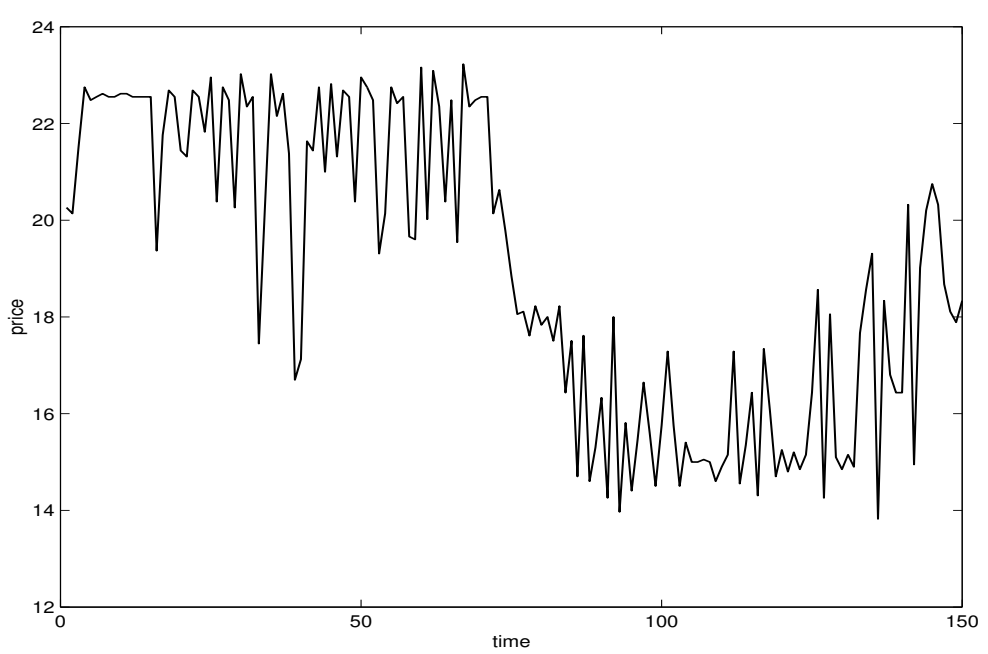

Figure 4: Simulated prices.

cartel and Walrasian equilibria occur approximately half of the time. In Figure 4 one can see that the cartel price prevails in the first half of the run, whereas the Walrasian price prevails in the second half.

Figures 1, 2, and 3 show an interesting feature, namely that the Walrasian equilibrium is played more frequently in the long-run than in the short-run. In the next section we provide theoretical evidence that the Walrasian equilibrium has, approximately, the largest basin of attraction when the error probabilities converge to zero. In fact, the Walrasian is the only state that has a basin of attraction in this limiting case.

\section{A Theoretical Analysis}

For each $\varepsilon$ and $\tilde{\varepsilon}$ the chain $Q^{\varepsilon, \tilde{\varepsilon}}$ is ergodic, hence there is a unique invariant probability measure. In line with other papers on stochastic stability (cf. Kandori et al. (1993), Young (1993), and Vega-Redondo (1997)) we are interested in the long-run behaviour of the dynamics when evolution has forced the probabilities of mistakes to zero. The standard argument for considering this limiting case is that firms learn to play the game better as time evolves. That is, we are interested in the (unique) invariant probability measure $\mu(\cdot)$ of the Markov chain with transition matrix $Q$, 
where ${ }^{3}$

$$
Q=\operatorname{limlim}_{\tilde{\varepsilon} \downarrow 0 \varepsilon \downarrow 0} Q^{\varepsilon, \tilde{\varepsilon}} .
$$

The support of $\mu(\cdot)$ constitutes the set of stochastically stable states. Note that, contrary to the standard literature we have two levels of evolution in this model. Due to the complexity of the combined dynamics, one cannot determine $\mu(\cdot)$ exactly. It turns out, however, that we can find an approximation, $\tilde{\mu}(\cdot)$, of this measure which can be analysed.

As a point of departure the first level of evolution, the quantity dynamics, is studied. For each $I=1, \ldots, N$, let $M_{I}=\lim _{\varepsilon \downarrow 0} M_{I}^{\varepsilon}$ be the limit Markov chain when the error in the quantity dynamics converges to zero. Note that $M_{I}$ has a unique invariant probability measure, say $\mu^{I}(\cdot)$. To facilitate further analysis it is assumed that for any vector of conjectures there is a unique equilibrium, i.e. a unique vector of quantities that solves eq. (1) for all firms. Furthermore, we assume that this equilibrium is an element of the quantity grid $\Gamma$.

Assumption 1 For all $\alpha \in \Lambda^{n}$ there exists a unique $q^{\alpha} \in \Gamma$ such that for all $i \in I_{n}$,

$$
P^{\prime}\left(q_{i}^{\alpha}+Q_{-i}^{\alpha}\right)\left(1+\alpha_{i}\right) \frac{n}{2} q_{i}^{\alpha}+P\left(q_{i}^{\alpha}+Q_{-i}^{\alpha}\right)-C^{\prime}\left(q_{i}^{\alpha}\right)=0 .
$$

Let the permutation on $\Gamma$ that corresponds to $q^{\alpha}$ be denoted by $k(I)$, i.e. $q(k(I))=$ $q^{\alpha}$. The following proposition states that for each vector of conjectures $\alpha(I)$ the unique stochastically stable state of the quantity dynamics is given by $q^{\alpha(I)}$.

Theorem 1 Let $I \in\{1, \ldots, N\}$ be given. Under Assumption 1, the unique invariant probability measure $\mu^{I}(\cdot)$ of the Markov chain with transition matrix $M_{I}$ is such that

$$
\mu^{I}\left(q^{\alpha(I)}\right)=1 .
$$

Proof. The proposition is proved using the theory developed by Milgrom and Roberts (1991). First note that for all $i \in I_{n}, \Gamma_{i}$ is a compact subset of $\mathbb{R}_{+}$. Define for all $i \in I_{n}$ the (continuous) function $\tilde{\pi}_{i}: \mathbb{R}_{+} \times \mathbb{R}_{+}^{n-1} \rightarrow \mathbb{R}_{+}$, given by

$$
\tilde{\pi}_{i}\left(q_{i}, q_{-i}\right)=-\left|P^{\prime}\left(q_{i}+Q_{-i}\right)\left(1+\alpha_{i}(I)\right) \frac{n}{2} q_{i}+P\left(q_{i}+Q_{-i}\right)-C^{\prime}\left(q_{i}\right)\right| .
$$

Consider the normal-form game $\left\langle I_{n},\left(\Gamma_{i}\right)_{i \in I_{n}},\left(\tilde{\pi}_{i}\right)_{i \in I_{n}}\right\rangle$. Let $S \subset \Gamma$, denote by $S_{i}$ the projection of $S$ on $\Gamma_{i}$ and define $S_{-i}=\prod_{j \neq i} S_{j}$. For all $i \in I_{n}$ the set of undominated strategies with respect to $S$ is given by the set

$$
U_{i}(S)=\left\{q_{i} \in \Gamma_{i} \mid \forall \forall_{y \in S_{i}} \exists_{q_{-i} \in S_{-i}}: \tilde{\pi}_{i}\left(q_{i}, q_{-i}\right) \geq \tilde{\pi}_{i}\left(y, q_{-i}\right)\right\} .
$$

\footnotetext{
${ }^{3}$ The order of limits is crucial in the results to be proved. First evolution drives the error probability of the quantity dynamics to zero and after that the error probability for the conjecture dynamics. Since it is assumed that quantity adaptation takes places more frequent than conjecture adaptation $(\tilde{p}<p)$, this is a straightforward assumption.
} 
Let $U(S)=\prod_{i \in I_{n}} U_{i}(S)$, the $k$-th iterate of which is given by $U^{k}(S)=U\left(U^{k-1}(S)\right)$, $k \geq 2$, where $U^{1}(S)=U(S)$. Note that since $q^{\alpha(I)}$ is unique we have

$$
U^{\infty}(\Gamma)=\left\{q^{\alpha(I)}\right\}
$$

Following Milgrom and Roberts (1991) we say that $\left\{q^{t}\right\}_{t \in \mathbb{N}}$ is consistent with adaptive learning if

$$
\forall_{\hat{t} \in \mathbf{N}} \exists_{\bar{t}>\hat{t}} \forall_{\tilde{t} \geq \bar{t}}: q^{\tilde{t}} \in U\left(\left\{q^{s} \mid \hat{t} \leq s<\tilde{t}\right\}\right) .
$$

Let $\hat{t} \in \mathbb{N}$, take $\bar{t}=\hat{t}+1$ and let $\tilde{t}=\bar{t}+k$ for some $k \in\{0,1,2, \ldots\}$. Then

$$
\left\{q^{s} \mid \hat{t} \leq s<\tilde{t}\right\}=\left\{q^{s} \mid s=\hat{t}, \ldots, \bar{t}+k-1\right\} .
$$

Let $\left\{q^{t}\right\}_{t \in \mathrm{N}}$ be generated by the pure quantity dynamics, i.e. the quantity dynamics without the experimentation (or mutation) part. Then we have by definition

$$
\forall y \in \Gamma_{i}: \tilde{\pi}_{i}\left(q_{i}^{\tilde{t}}, q_{-i}^{\tilde{t}-1}\right) \geq \tilde{\pi}_{i}\left(y, q_{-i}^{\tilde{t}-1}\right)
$$

Furthermore, it holds that $q^{\tilde{t}-1} \in\left\{q^{s} \mid \bar{t} \leq s<\tilde{t}\right\}$. Hence, we can conclude that $\left\{q^{t}\right\}_{t \in \mathbf{N}}$ is consistent with adaptive learning. From Milgrom and Roberts (1991, Theorem 7) one obtains that $\left\|q^{t}-q^{\alpha(I)}\right\| \rightarrow 0$ as $t \rightarrow \infty$. Since $\Gamma$ is finite we have

$$
\exists_{\bar{t} \in \mathbf{N}} \forall_{t \geq \bar{t}}: q^{t}=q^{\alpha(I)} .
$$

So, $\left\{q^{\alpha(I)}\right\}$ is the only recurrent state of the (mutation-free) pure quantity dynamics. From Young (1993) we know that the stochastically stable states are among the recurrent states of the mutation-free dynamics. Hence, $\mu^{I}\left(q^{\alpha(I)}\right)=1$.

Before we turn to Theorem 2, the following lemma is introduced, which plays a pivotal role its proof. It compares the equilibrium profits for different conjectures. Suppose that the market is in a monomorphic state, i.e. all firms have the same conjecture. The question is what happens to equilibrium profits if $k$ firms deviate to another conjecture. If $n-k$ firms have a conjecture equal to $\alpha$ and $k$ firms have a conjecture equal to $\alpha^{\prime}$, let the (unique) equilibrium quantities be denoted by $q_{k}^{\alpha}$ and $q_{k}^{\alpha^{\prime}}$, respectively.

Lemma 1 For all $k \in\{1,2, \ldots, n-1\}$ and $\alpha>\alpha^{\prime}$ it holds that

$$
P\left((n-k) q_{k}^{\alpha}+k q_{k}^{\alpha^{\prime}}\right) q_{k}^{\alpha^{\prime}}-C\left(q_{k}^{\alpha^{\prime}}\right)>P\left((n-k) q_{k}^{\alpha}+k q_{k}^{\alpha^{\prime}}\right) q_{k}^{\alpha}-C\left(q_{k}^{\alpha}\right)
$$

The proof of this lemma can be found in Appendix B. Lemma 1 plays a similar role as the claim in Vega-Redondo (1997, p. 381). The main result in that paper is driven by the fact that if at least one firm plays the Walrasian quantity against the other firms playing another quantity, the firm with the Walrasian quantity has a strictly 
higher profit. In our model the dynamics is more elaborate. Suppose that all firms have the Walrasian conjecture and that the quantity dynamics is in equilibrium, i.e. the Walrasian equilibrium. If at least one player has another conjecture not only its own equilibrium quantity changes, but also the equilibrium quantities of the firms that still have the Walrasian conjecture. Lemma 1 states that the firms with the lower conjecture still have the highest equilibrium profit. This is intuitively clear form the first-order condition (1). The firms with the lower conjecture increase their production until the difference between the price and the marginal costs reaches a lower, but positive, level than the firms with the higher conjecture. Therefore, the total profit of having a lower conjecture is higher. This happens because the firms do not realise that in the future their behaviour will be imitated by other firms which puts downward pressure on industry profits.

Some additional notation and assumptions are needed in the following. For a matrix $A$ let $\lambda_{j}(A)$ denote the $j$-th largest eigenvalue in absolute value of $A$. Furthermore, define $\lambda_{k}(I, J)=\lim _{\tilde{\varepsilon} \downarrow 0} \lambda_{k}^{\tilde{\varepsilon}}(I, J)$ and let $\zeta=\max _{k_{I}}\left\{\sum_{K \neq I} \sum_{l=1}^{m} Q_{k_{I} l_{K}}\right\}$. The following assumptions are made.

Assumption 2 All elementary divisors of $Q$ are linear.

Assumption $3 \zeta<\frac{1}{2}\left[1-\max _{I \in\{1, \ldots, N\}} \lambda_{2}\left(M_{I}\right)\right]$.

Since the probability measures $\nu_{i}(\cdot)$ and $\tilde{\nu}_{i}(\cdot)$ have full support for all $i \in I_{n}$, all eigenvalues of $Q$ will generically be distinct and, hence, Assumption 2 will generically be satisfied. Let $\alpha(1)$ be the monomorphic state where all firms have the Walrasian conjecture, i.e. $\alpha(1)=(-1, \ldots,-1)$ We can now state the following theorem.

Theorem 2 Suppose that Assumptions 1-3 hold. Then there exists an ergodic Markov chain on $\Lambda^{n}$ with transition matrix $\tilde{Q}$ and unique invariant probability measure $\tilde{\mu}(\cdot)$. For $\tilde{\mu}(\cdot)$ it holds that $\tilde{\mu}\left(q^{\alpha(1)}\right)=1$. Furthermore, $\tilde{\mu}(\cdot)$ is an approximation of $\mu(\cdot)$ of order $O(\zeta)$.

Proof. The approximation result follows from the theory of nearly-complete decomposability (cf. Courtois (1977) and Appendix A). First, define

$$
Q^{*}=\left[\begin{array}{cccc}
M_{1} & 0 & \ldots & 0 \\
0 & \ddots & \ddots & \vdots \\
\vdots & \ddots & \ddots & 0 \\
0 & \ldots & 0 & M_{N}
\end{array}\right],
$$


and take the matrix $C$ such that

$$
\zeta C_{k_{I} l_{J}}= \begin{cases}\lambda_{k}(I, J) M_{I}(k, l) & \text { if } I \neq J \\ -\sum_{K \neq I} \lambda_{k}(I, K) M_{I}(k, l) & \text { if } I=J\end{cases}
$$

Note that $Q=Q^{*}+\zeta C$. So, the transition matrix $Q$ has been decomposed into a block diagonal matrix $Q^{*}$, where each diagonal block is the transition matrix for the quantity dynamics of a given vector of conjectures, and a matrix that reflects the conjecture dynamics. The constant $\zeta$ can be interpreted as the maximum degree of coupling between subsystems $M_{I}$.

Given the result of Theorem 1 one can aggregate $Q$ using $\mu^{I}(\cdot)$ in the following way. Define a Markov chain on $\Lambda^{n}$ with transition matrix $\tilde{Q}$ which has typical element

$$
\begin{aligned}
\tilde{Q}(I, J) & =\sum_{k=1}^{m} \mu^{I}(q(k)) \sum_{l=1}^{m} Q_{k_{I} l_{J}} \\
& =\sum_{k=1}^{m} \mu^{I}(q(k)) \lambda_{k}(I, J) \sum_{l=1}^{m} M_{I}(k, l) \\
& =\sum_{k=1}^{m} \mu^{I}(q(k)) \lambda_{k}(I, J)=\lambda_{k(I)}(I, J) .
\end{aligned}
$$

Note that the transition matrix $\tilde{Q}$ is the limit of a sequence of ergodic Markov chains with transition matrices $\tilde{Q}^{\tilde{\varepsilon}}$ with $\tilde{Q}^{\tilde{\varepsilon}}(I, J)=\lambda_{k(I)}^{\tilde{\varepsilon}}(I, J)$. So, $\tilde{Q}$ has a unique invariant probability measure $\tilde{\mu}(\cdot)$. Under Assumptions 2 and 3 the matrix $Q$ is nearly-completely decomposable. From Courtois (1977, Section 3.2) this directly implies that $\tilde{\mu}(\cdot)$ is an $O(\zeta)$ approximation of $\mu(\cdot)$.

The result on $\tilde{\mu}(\cdot)$ is obtained by using the techniques developed by Freidlin and Wentzell (1984). First we establish the set of recurrent states for the mutation-free dynamics of $\tilde{Q}^{\tilde{\varepsilon}}$. This is the dynamics without the experimentation part and is thus equal for all $\tilde{\varepsilon}>0$. From (3) one can see that the transition probabilities for this dynamics are equal to the transition probabilities of going from one vector of conjectures $\alpha(I)$ to another vector $\alpha(J)$ given that the current quantity vector is the equilibrium $q^{\alpha(I)}$. So, the dynamics of $\tilde{Q}^{\tilde{\varepsilon}}$ is the pure conjecture dynamics if the quantity dynamics gets sufficient time to settle in equilibrium. Let the transition matrix for this aggregated pure conjecture dynamics be denoted by $\tilde{Q}_{0}$.

Lemma 2 The set $\mathcal{A}$ of recurrent states for the aggregated mutation-free conjecture dynamics with transition matrix $\tilde{Q}_{0}$ is given by the set of monomorphic states, i.e.

$$
\mathcal{A}=\{\{(\alpha, \ldots, \alpha)\} \mid \alpha \in \Lambda\} .
$$


The proof of this lemma can be found in Appendix C. Define the costs between $\alpha(I)$ and $\alpha(J)$ to be

$$
c(\alpha(I), \alpha(J))=\min _{K=1, \ldots, N}\left\{d(\alpha(I), \alpha(K)) \mid \tilde{Q}_{0}(K, J)>0\right\},
$$

where $d(\alpha(I), \alpha(K))=\sum_{i \in I_{n}} \mathbb{1}_{\left(\alpha_{i}(I) \neq \alpha_{i}(K)\right)}$. The cost between $\alpha(I)$ and $\alpha(J)$ is the minimum number of mutations from $\alpha(I)$ that is needed for the pure conjecture dynamics to have positive probability of reaching $\alpha(J)$. Let $\alpha \in \Lambda^{n}$. An $\alpha$-tree $H_{\alpha}$ is a collection of ordered pairs $\left(\alpha^{\prime}, \alpha^{\prime \prime}\right)$ such that:

1. every $\alpha^{\prime} \in \Lambda^{n} \backslash\{\alpha\}$ is the first element of exactly one pair;

2. for all $\alpha^{\prime} \in \Lambda^{n} \backslash\{\alpha\}$ there exists a path $\left(\alpha^{\prime}, \alpha^{1}\right),\left(\alpha^{1}, \alpha^{2}\right), \ldots,\left(\alpha^{s-1}, \alpha^{s}\right),\left(\alpha^{s}, \alpha\right)$ in $H_{\alpha}$.

For each $\alpha$-tree $H_{\alpha}$ the cost of tree $H_{\alpha}$ is defined by

$$
c\left(H_{\alpha}\right)=\sum_{\left(\alpha^{\prime}, \alpha^{\prime \prime}\right) \in H_{\alpha}} c\left(\alpha^{\prime}, \alpha^{\prime \prime}\right) .
$$

First, we build an $\alpha(1)$-tree $H^{*}$ with minimal costs. Then it is shown that for any state $\alpha \in \mathcal{A} \backslash\{\alpha(1)\}$ and any $\alpha$-tree $H_{\alpha}$ the costs will be higher. From Freidlin and Wentzell (1984, Lemma 6.3.1) one can then conclude that $\alpha(1)$ is the unique element in the support of $\tilde{\mu}(\cdot)$. Young (1993) has shown that the minimum cost tree is among the $\alpha$-trees where $\alpha$ is an element of a recurrent class of the mutation-free dynamics. Thus, from Lemma 2 we know that we only need to consider the monomorphic states in $\mathcal{A}$. This implies that for all $\alpha$-trees $H_{\alpha}, \alpha \in \mathcal{A}$, we have $c\left(H_{\alpha}\right) \geq|\mathcal{A}|-1$, since one always needs at least one experiment to leave a monomorphic state.

Consider $\alpha(1)$ and the $\alpha(1)$-tree $H^{*}$ that is constructed in the following way. Let $\alpha \in \mathcal{A} \backslash\{\alpha(1)\}$. For all $i \in I_{n}$ we have $\alpha_{i}>\alpha_{i}(1)$. Suppose that one firm $i$ experiments to $\alpha_{i}(1)=-1$, while the other firms cannot revise their output. According to Lemma 1 with $k=1$ this firm has a higher profit in quantity equilibrium than the other firms. If one period later all other firms $j \neq i$ get the opportunity to revise their conjectural variation (which happens with positive probability) they will all choose $\alpha_{j}(1)=-1$. Hence, one mutation suffices to reach $\alpha(1)$ and therefore $c\left(H^{*}\right)=|\mathcal{A}|-1$.

Conversely, let $H_{\alpha}$ be an $\alpha$-tree for some $\alpha \in \mathcal{A} \backslash\{\alpha(1)\}$. Then somewhere in this tree there is a path between $\alpha(1)$ and a monomorphic state $\alpha^{\prime}$ with $\alpha_{i}^{\prime}>-1$ for all $i \in I_{n}$. Suppose that starting from $\alpha(1)$ one firm $i$ experiments to $\alpha_{i}^{\prime}$. From Lemma 1 with $k=n-1$ it is obtained that firm $i$ has a strictly lower profit than the other firms in quantity equilibrium. So, to drive the system away from $\alpha(1)$ to $\alpha^{\prime}$ at least two mutations are needed. Hence, $c\left(H_{\alpha}\right)>c\left(H^{*}\right)$. 
Theorem 2 gives a result on the convergence of market interaction to the Walrasian equilibrium that is similar to the result of Vega-Redondo (1997). Apparently, profit imitation is such a strong force that it also drives this more elaborate behavioural model to the Walrasian equilibrium. Note, however, that the result in Theorem 2 is an approximation. It might well be that the support of $\mu(\cdot)$ consists of more states than just the Walrasian equilibrium. This is actually suggested by the simulations in Section 3.

A crucial assumption is the one on the maximum degree of coupling between subsystems $M^{I}, \zeta$, as stated in Assumption 3. This parameter should not be too large. Intuitively, this condition requires that the interaction between subsystems $M^{I}$ is sufficiently low, i.e. that the conjecture dynamics does not happen too frequent. In Proposition 1 a sufficient condition on $\tilde{p}$ is given for Assumption 3 to hold.

Proposition 1 If $\tilde{p}<1-\left(\frac{3}{4}\right)^{1 / n}$, then Assumption 3 is satisfied.

Proof. Let $I \in\{1,2, \ldots, N\}$. From Bauer et al. (1969) we obtain an upper bound for the second largest eigenvalue of $M^{I}$ :

$$
\begin{gathered}
\lambda_{2}\left(M^{I}\right) \leq \min \left\{\max _{1 \leq \theta, \rho \leq m} \frac{1}{2} \sum_{i=1}^{m} v_{i}^{1}\left(M^{I}\right)\left|\frac{M^{I}(i, \theta)}{v_{\theta}^{1}\left(M^{I}\right)}-\frac{M^{I}(i, \rho)}{v_{\rho}^{1}\left(M^{I}\right)}\right|,\right. \\
\left.\max _{1 \leq \theta, \rho \leq m} \frac{1}{2} \sum_{i=1}^{m}\left|M^{I}(\theta, i)-M^{I}(\rho, i)\right|\right\},
\end{gathered}
$$

where $v^{1}\left(M^{I}\right)$ is the eigenvector corresponding to the largest eigenvalue of $M^{I}$. Since $M^{I}$ is a stochastic matrix we have that

$$
v_{i}^{1}\left(M^{I}\right)=\mu_{i}^{I}=\mathbb{1}_{\left(q=q^{\alpha(I)}\right)} .
$$

Consider the first term on the right hand side of (6). For $\theta=k(I)$ and $\rho \neq k(I)$, we get

$$
\begin{aligned}
& \frac{1}{2} \sum_{i=1}^{m} v_{i}^{1}\left(M^{I}\right)\left|\frac{M^{I}(i, k(I))}{v_{k(I)}^{1}\left(M^{I}\right)}-\frac{M^{I}(i, \rho)}{v_{\rho}^{1}\left(M^{I}\right)}\right| \\
= & \frac{1}{2}\left|\frac{M^{I}(k(I), k(I))}{\mu_{k(I)}^{I}}-\frac{M^{I}(k(I), \rho)}{\mu_{\rho}^{I}}\right| \\
= & \infty
\end{aligned}
$$

since $\mu^{I}(q(\rho))=0$ and $\frac{M^{I}(k(I), k(I))}{\mu_{k(I)}^{I}}=M^{I}(k(I), k(I))=1$.

The maximum of the second term on the right hand side of (6) is attained for $\theta=k(I)$ and some $\rho \neq k(I)$, such that $q(k(I))$ is not a best response to $q(\rho)$. One 
obtains that

$$
\frac{1}{2} \sum_{i=1}^{m}\left|M^{I}(k(I), i)-M^{I}(\rho, i)\right| \leq \frac{1}{2}\left|M^{I}(k(I), k(I))\right|=\frac{1}{2},
$$

since $q(k(I))$ is a best response to $q(k(I))$. Hence, we find that $\lambda_{2}\left(M^{I}\right) \leq \frac{1}{2}$ for all $I=1, \ldots, N$. So, we have that

$$
\frac{1}{2}\left[1-\max _{I=1, \ldots, N} \lambda_{2}\left(M^{I}\right)\right] \geq \frac{1}{4} .
$$

Note that it holds that

$$
\begin{aligned}
\zeta & =\max _{k_{I}}\left\{\sum_{K \neq I} \sum_{l=1}^{m} Q_{k_{I} l_{K}}\right\} \\
& =\max _{k_{I}}\left\{\sum_{K \neq I} \lambda_{k}(I, K)\right\} \\
& =\max _{k_{I}}\left\{1-\lim _{\tilde{\varepsilon} \downarrow 0} \lambda_{k}^{\tilde{\varepsilon}}(I, I)\right\} .
\end{aligned}
$$

Furthermore, by definition we have that

$$
\lambda_{k}^{\tilde{\varepsilon}}(I, I) \geq \prod_{i \in I_{n}}\left\{(1-\tilde{\varepsilon})(1-\tilde{p})+\tilde{\varepsilon} \tilde{\nu}_{i}\left(\alpha_{i}(I)\right)\right\} .
$$

Therefore, we conclude that

$$
\begin{aligned}
\zeta & \leq 1-\lim _{\tilde{\varepsilon} \downarrow 0} \prod_{i \in I_{n}}\left\{(1-\tilde{\varepsilon})(1-\tilde{p})+\tilde{\varepsilon} \tilde{\nu}_{i}\left(\alpha_{i}(I)\right)\right\} \\
& =1-(1-\tilde{p})^{n}<\frac{1}{4} \\
\Longleftrightarrow \tilde{p} & <1-\left(\frac{3}{4}\right)^{1 / n}
\end{aligned}
$$

which proves the proposition.

\section{Discussion}

The model presented in this chapter extends existing evolutionary models of e.g. Vega-Redondo (1997), Schenk-Hoppé (2000) and Schipper (2003) by allowing for dynamics at two levels. We model quantity dynamics based on myopic optimisation by firms that includes the conjectured market response to the firm's own quantitysetting behaviour which is modelled by means of a conjecture parameter. At a second level, we allow firms to change or adapt their behaviour in the sense that they can change their conjecture. This decision is also modelled to be boundedly 
rational. Firms look at their competitors and imitate the behaviour of the most successful firm.

The main conclusion of Theorem 2 is that if behavioural adjustment takes place at a sufficiently lower rate than quantity adjustment, the market ends up in the Walrasian equilibrium in the long-run. To be more precise, the Walrasian equilibrium is the only outcome that will be observed a significant amount of time in the long-run. A sufficient condition for this result to hold is that the conjecture dynamics occurs at a sufficiently low frequency. A simulation study shows that in the long-run also the cartel and Cournot-Nash equilibria can arise at a significant frequency. The main point of Theorem 2 is, however, that even with more elaborate behavioural dynamics than e.g. Vega-Redondo (1997), evolution still selects the Walrasian equilibrium. The appeal of this equilibrium lies in the fact that if behaviour is guided by profit imitation, i.e. relative payoffs, this leads to spitefulness in a firm's actions. This in turn leads to selection of the Walrasian equilibrium.

An important feature of our model that triggers the result of Theorem 2 is the fact that we model an explicit dynamic process where firms learn from the past. This induces them to adapt their behaviour if their profit falls behind their competitors' profits. This contrasts, for example, standard repeated games where time plays an implicit role. To quote Vives (1999): in a "pure repeated game framework[...]history matters only because firms threaten it to matter". Therefore, a cartel outcome can be sustained as an equilibrium in such models. The combination of time having an explicit role and boundedly rational firms has important consequences for the long-run outcome of market interaction since it avoids folk theorem-like results and instead pins down a unique equilibrium outcome.

\section{Appendix}

\section{A Nearly-Complete Decomposability}

This appendix is based on Courtois (1977). Intuitively, a nearly-complete decomposable system is a Markov chain where the matrix of transition probabilities can be divided into blocks such that the interaction between blocks is small relative to interaction within blocks. In the remainder let $Q$ be an $n \times n$ irreducible stochastic matrix. The dynamic process $\left(y_{t}\right)_{t \in \mathbb{N}}$, where $y_{t} \in \mathbb{R}^{n}$ for all $t \in \mathbb{N}$, is then given by

$$
\left(y_{t+1}\right)^{\top}=\left(y_{t}\right)^{\top} Q .
$$

Note that $Q$ can be written as follows:

$$
Q=Q^{*}+\zeta C,
$$


where $Q^{*}$ is of order $n$ and given by

$$
Q^{*}=\left[\begin{array}{ccccc}
Q_{1}^{*} & & & & \\
& \ddots & & 0 & \\
& & Q_{I}^{*} & & \\
& 0 & & \ddots & \\
& & & & Q_{N}^{*}
\end{array}\right] .
$$

The matrices $Q_{I}^{*}, I=1, \ldots, N$, are irreducible stochastic matrices of order $n(I)$. Hence $n=\sum_{I=1}^{N} n(I)$. Therefore the sums of the rows of $C$ are zero. We choose $\zeta$ and $C$ such that for all rows $k_{I}, I=1, \ldots, N, k=1, \ldots, n$, it holds that

$$
\zeta \sum_{J \neq I} \sum_{l=1}^{n(J)} C_{k_{I} l_{J}}=\sum_{J \neq I} \sum_{l=1}^{n(J)} Q_{k_{I} l_{J}}
$$

and

$$
\zeta=\max _{k_{I}}\left(\sum_{J \neq I} \sum_{l=1}^{n(J)} Q_{k_{I} l_{J}}\right)
$$

where the $k_{I}$ denotes the $k$-th element in the $I$-th block. The parameter $\zeta$ is called the maximum degree of coupling between subsystems $Q_{I}^{*}$.

It is assumed that all elementary divisors ${ }^{4}$ of $Q$ and $Q^{*}$ are linear. Then the spectral density composition of the $t$-step probabilities $-Q^{t}$ - can be written as

$$
Q^{t}=\sum_{I=1}^{N} \sum_{k=1}^{n(I)} \lambda^{t}\left(k_{I}\right) Z\left(k_{I}\right)
$$

where

$$
Z\left(k_{I}\right)=s\left(k_{I}\right)^{-1} v\left(k_{I}\right) v\left(k_{I}\right)^{\top}
$$

$\lambda\left(k_{I}\right)$ is the $k_{I}$-th maximal eigenvalue in absolute value of $Q, v\left(k_{I}\right)$ is the corresponding eigenvector normalised to one using the vector norm $\|\cdot\|_{1}$, and $s\left(k_{I}\right)$ is the condition number $s\left(k_{I}\right)=v\left(k_{I}\right)^{\top} v\left(k_{I}\right)$. Since $Q$ is a stochastic matrix, the PerronFrobenius theorem gives that the maximal eigenvalue of $Q$ equals 1. Therefore, (A.3) can be rewritten as

$$
Q^{t}=Z\left(1_{1}\right)+\sum_{I=2}^{N} \lambda^{t}\left(1_{I}\right) Z\left(1_{I}\right)+\sum_{I=1}^{N} \sum_{k=2}^{n(I)} \lambda^{t}\left(k_{I}\right) Z\left(k_{I}\right) .
$$

\footnotetext{
${ }^{4}$ See e.g. Lancaster and Tismentetsky (1985).
} 
If one defines for each matrix $Q_{I}^{*}$ in a similar way $Z^{*}\left(k_{I}\right), s^{*}\left(k_{I}\right), \lambda^{*}\left(k_{I}\right)$, and $v^{*}\left(k_{I}\right)$, e.g. $\lambda^{*}\left(k_{I}\right)$ is the $k$-th maximal eigenvalue in absolute value of $Q_{I}^{*}$, then one can find a similar spectral decomposition for $Q^{*}$, i.e.

$$
\left(Q^{*}\right)^{t}=\sum_{I=1}^{N} Z^{*}\left(1_{I}\right)+\sum_{I=1}^{N} \sum_{k=2}^{n(I)}\left(\lambda^{*}\right)^{t}\left(k_{I}\right) Z^{*}\left(k_{I}\right),
$$

using the fact that $v_{k_{I}}^{*}\left(1_{I}\right)=n(I)^{-1}$ for all $k_{I}$. The behaviour through time of $y_{t}$ and $y_{t}^{*}$, where the dynamics of $\left(y_{t}\right)_{t \in \mathbf{N}}$ is described by (A.1) and the process $\left(y_{t}^{*}\right)_{t \in \mathbf{N}}$ is defined by

$$
\left(y_{t+1}^{*}\right)^{\top}=\left(y_{t}^{*}\right)^{\top} Q^{*}
$$

are therefore also specified by (A.4) and (A.5). The behaviour of $y_{t}$ can be seen as long-run behaviour whereas $y_{t}^{*}$ describes short-run behaviour. The comparison between both processes follows from two theorems as proven by Simon and Ando (1961).

Theorem A.1 For an arbitrary positive real number $\xi$, there exists a number $\zeta_{\xi}$ such that for $\zeta<\zeta_{\xi}$,

$$
\max _{p, q}\left|Z_{p q}\left(k_{I}\right)-Z_{p q}^{*}\left(k_{I}\right)\right|<\xi
$$

for any $2 \leq k \leq n(I), 1 \leq I \leq N$, where $1 \leq p, q \leq n$.

Theorem A.2 For an arbitrary positive real number $\omega$, there exists a number $\zeta_{\omega}$ such that for $\zeta<\zeta_{\omega}$,

$$
\max _{k, l}\left|Z_{k_{I} l_{J}}\left(k_{I}\right)-v_{l_{J}}^{*}\left(1_{J}\right) \alpha_{I J}\left(1_{K}\right)\right|<\omega,
$$

for any $1 \leq k \leq n(I), 1 \leq l \leq n(J), 1 \leq K, I, J \leq N$, and where $\alpha_{I J}\left(1_{K}\right)$ is given by

$$
\alpha_{I J}\left(1_{K}\right)=\sum_{k=1}^{n(I)} \sum_{l=1}^{n(J)} v_{k_{I}}^{*} z_{k_{I} l_{J}}\left(1_{K}\right) .
$$

It can be shown that for all $I=1, \ldots, N, \lambda\left(1_{I}\right)$ is close to unity. Therefore $\lambda^{t}\left(1_{I}\right)$ will also be close to unity for small $t$. Hence, the first two terms on the right-hand side of eq. (A.4) will not vary much for $t<T_{2}$, for some $T_{2}>0$. The first term of the right-hand-side of (A.5) does not change at all. Hence, for $t<T_{2}$ the behaviour through time of $y_{t}$ and $y_{t}^{*}$ is determined by the last terms of $Q^{t}$ and $\left(Q^{*}\right)^{t}$, respectively. Also, if $\varepsilon \rightarrow 0$ it can be shown that $\lambda\left(k_{I}\right) \rightarrow \lambda^{*}\left(k_{I}\right)$ and from Theorem A.1 it follows that $Z\left(k_{I}\right) \rightarrow Z^{*}\left(k_{I}\right)$, for all $k=2, \ldots, n(I)$ and $I=1, \ldots, N$. This means that for $\zeta$ small and $t<T_{2}$ the paths of $y_{t}$ and $y_{t}^{*}$ are very close. 
The eigenvalues $\lambda^{*}\left(k_{I}\right)$ are strictly less than unity in absolute value for all $k=$ $2, \ldots, n(I)$, and $I=1, \ldots, N$. For any positive real number $\xi_{1}$ we can therefore define a smallest time $T_{1}^{*}$ such that

$$
\max _{1 \leq p, q \leq n}\left|\sum_{I=1}^{N} \sum_{k=2}^{n(I)}\left(\lambda^{*}\right)^{t}\left(k_{I}\right) Z_{p q}^{*}\left(k_{I}\right)\right|<\xi_{1} \quad \text { for } t>T_{1}^{*} .
$$

Similarly we can find a $T_{1}$ such that

$$
\max _{1 \leq p, q \leq n}\left|\sum_{I=1}^{N} \sum_{k=2}^{n(I)} \lambda^{t}\left(k_{I}\right) Z_{p q}\left(k_{I}\right)\right|<\xi_{1} \quad \text { for } t>T_{1} .
$$

Theorem A.1 plus convergence of the eigenvalues with $\zeta$ then ensures that $T_{1} \rightarrow T_{1}^{*}$ as $\zeta \rightarrow 0$. We can always choose $\zeta$ such that $T_{2}>T_{1}$. As long as $\zeta$ is not identical to zero it holds that $\lambda\left(1_{I}\right)$ is not identical to unity for $I=2, \ldots, N .{ }^{5}$ Therefore, there will be a time $T_{3}>0$ such that for sufficiently small $\xi_{3}$,

$$
\max _{1 \leq p, q \leq n}\left|\sum_{I=1}^{N} \sum_{k=2}^{n(I)} \lambda^{t}\left(1_{I}\right) Z_{p q}\left(1_{I}\right)\right|<\xi_{3} \quad \text { for } t>T_{3} .
$$

This implies that for $T_{2}<t<T_{3}$, the last term of $Q^{t}$ is negligible and the path of $y_{t}$ is determined by the first two components of $Q^{t}$. According to Theorem A.2 it holds that for any $I$ and $J$ the elements of $Z\left(1_{K}\right)$,

$$
Z_{k_{I} 1_{J}}\left(1_{K}\right), \ldots, Z_{k_{I} l_{J}}\left(1_{K}\right), \ldots, Z_{k_{I} n(J)_{J}}\left(1_{K}\right),
$$

depend essentially on $I, J$ and $l$, and are almost independent of $k$. So, for any $I$ and $J$ they are proportional to the elements of the eigenvector of $Q_{J}^{*}$ corresponding to the largest eigenvalue. Since $Q^{*}$ is stochastic and irreducible, this eigenvector corresponds to the unique invariant probability measure $\mu_{J}^{*}$ of the Markov chain with transition matrix $Q_{J}^{*}$. Thus, for $T_{2}<t<T_{3}$ the elements of the vector $y_{t},\left(y_{l_{J}}\right)_{t}$, will approximately have a constant ratio that is similar to that of the elements of $\mu_{J}^{*}$. Finally, for $t>T_{3}$ the behaviour of $y_{t}$ is almost completely determined by the first term of $Q^{t}$. So, $y_{t}$ evolves towards $v\left(1_{1}\right)$, which corresponds to the unique invariant probability measure $\mu$ of the Markov chain with transition matrix $Q$. Summarising, the dynamics of $y_{t}$ can be described as follows.

1. Short-run dynamics: $t<T_{1}$. The predominant terms in $Q^{t}$ and $\left(Q^{*}\right)^{t}$ are the last ones. Hence, $y_{t}$ and $y_{t}^{*}$ evolve similarly.

2. Short-run equilibrium: $T_{1}<t<T_{2}$. The last terms of $Q^{t}$ and $\left(Q^{*}\right)^{t}$ have vanished while for all $I, \lambda^{t}\left(1_{I}\right)$ remains close to unity. A similar equilibrium is therefore reached within each subsystem of $Q$ and $Q^{*}$.

\footnotetext{
${ }^{5}$ If $\zeta=0$, all blocks $Q_{I}$ are irreducible and then we would have $\lambda\left(1_{I}\right)=\lambda^{*}\left(1_{I}\right)=1$ for all $I$.
} 
3. Long-run dynamics: $T_{2}<t<T_{3}$. The predominant term in $Q^{t}$ is the second one. The whole system moves to equilibrium, while the short-run equilibria in the subsystems are approximately maintained.

4. Long-run equilibrium: $t>T_{3}$. The first term of $Q^{t}$ dominates. Therefore, a global equilibrium is attained.

The above theory implies that one can estimate $\mu(\cdot)$ by calculating $\mu_{I}^{*}$ for $I=$ $1, \ldots, N$, and the invariant measure $\tilde{\mu}$ of the process

$$
\left(\tilde{y}_{t+1}\right)^{\top}=\left(\tilde{y}_{t}\right)^{\top} P
$$

where $\left(\tilde{y}_{I}\right)_{t}=\sum_{k=1}^{n(I)}\left(y_{k_{I}}\right)_{t}$ for all $I=1, \ldots, N$, and some transition matrix $P$. For $t>T_{2}$ we saw that $\frac{\left(y_{k_{I}}\right)_{t}}{\left(\tilde{y}_{I}\right)_{t}} \approx \mu_{I, k}^{*}$. Hence, the probability of a transition from group $I$ to group $J$ is given by

$$
\left(p_{I J}\right)_{t+1}=\left(\tilde{y}_{I}\right)_{t}^{-1} \sum_{k=1}^{n(I)}\left(y_{k_{I}}\right)_{t} \sum_{l=1}^{n(J)} Q_{k_{I} l_{J}} .
$$

For $t>T_{2}$ this can be approximated by

$$
\left(p_{I J}\right)_{t+1} \approx \sum_{k=1}^{n(I)} \mu_{I, k}^{*} \sum_{l=1}^{n(J)} Q_{k_{I} l_{J}} \equiv p_{I J} .
$$

So, by taking $P=\left[p_{I J}\right]$, the process in (A.7) gives a good approximation for $t>T_{2}$ of the entire process $\left(y_{t}\right)_{t \in \mathbb{N}}$. It is shown in Courtois (1977, Section 2.1) that the error of this approximation is of order $O(\zeta)$.

Until now we have not been concerned by how large $\zeta$ can be. It was stated that for $T_{1}^{*}<t<T_{2}$, the original system $Q$ is in a short-run equilibrium close to the equilibrium of the completely decomposable system $Q^{*}$. If this is to occur it must hold that $T_{1}^{*}<T_{2}$. Every matrix $Q$ can be written in the form of eq. (A.2), but not for all matrices it holds that $T_{1}^{*}<T_{2}$. Systems that satisfy the condition $T_{1}^{*}<T_{2}$ are called nearly-complete decomposable systems (cf. Ando and Fisher (1963)). Since $T_{1}^{*}$ is independent of $\zeta$ and $T_{2}$ increases with $\zeta \rightarrow 0$, the condition is satisfied for $\zeta$ sufficiently small. It is shown in Courtois (1977, Section 3.2) that a sufficient condition for nearly-complete decomposability is given by

$$
\zeta<\frac{1}{2}\left[1-\max _{I=1, \ldots, N}\left|\lambda^{*}\left(2_{I}\right)\right|\right] .
$$

\section{B Proof of Lemma 1}

Since all firms are identical and solutions to the first-order conditions are unique, firms with the same conjecture have the same equilibrium quantity. Therefore, the 
equilibrium quantities $q_{k}^{\alpha}$ and $q_{k}^{\alpha^{\prime}}$ satisfy

$$
\begin{aligned}
P^{\prime}\left((n-k) q_{k}^{\alpha}+k q_{k}^{\alpha^{\prime}}\right)(1+\alpha) \frac{n}{2} q_{k}^{\alpha}+P\left((n-k) q_{k}^{\alpha}+k q_{k}^{\alpha^{\prime}}\right)-C^{\prime}\left(q_{k}^{\alpha}\right) & =0 \\
P^{\prime}\left((n-k) q_{k}^{\alpha}+k q_{k}^{\alpha^{\prime}}\right)\left(1+\alpha^{\prime}\right) \frac{n}{2} q_{k}^{\alpha^{\prime}}+P\left((n-k) q_{k}^{\alpha}+k q_{k}^{\alpha^{\prime}}\right)-C^{\prime}\left(q_{k}^{\alpha^{\prime}}\right) & =0 .
\end{aligned}
$$

These first-order conditions imply that

$$
\begin{aligned}
P^{\prime}\left((n-k) q_{k}^{\alpha}+k q_{k}^{\alpha^{\prime}}\right) & (1+\alpha) \frac{n}{2} q_{k}^{\alpha}-C^{\prime}\left(q_{k}^{\alpha}\right) \\
& =P^{\prime}\left((n-k) q_{k}^{\alpha}+k q_{k}^{\alpha^{\prime}}\right)\left(1+\alpha^{\prime}\right) \frac{n}{2} q_{k}^{\alpha^{\prime}}-C^{\prime}\left(q_{k}^{\alpha^{\prime}}\right) .
\end{aligned}
$$

Suppose that $q_{k}^{\alpha} \geq q^{\alpha^{\prime}}$. There are two possible cases:

1. if $C^{\prime}\left(q_{k}^{\alpha}\right) \geq C^{\prime}\left(q_{k}^{\alpha^{\prime}}\right)$, then (B.1) immediately gives a contradiction;

2. if $C^{\prime}\left(q_{k}^{\alpha}\right)<C^{\prime}\left(q_{k}^{\alpha^{\prime}}\right)$, then according to (B.1) it should hold that

$$
-P^{\prime}\left((n-k) q_{k}^{\alpha}+k q_{k}^{\alpha^{\prime}}\right)(1+\alpha) \frac{n}{2} q_{k}^{\alpha} \leq P^{\prime}\left((n-k) q_{k}^{\alpha}+k q_{k}^{\alpha^{\prime}}\right)\left(1+\alpha^{\prime}\right) \frac{n}{2} q_{k}^{\alpha^{\prime}} .
$$

This implies that $\frac{q_{k}^{\alpha}}{q_{k}^{\alpha^{\prime}}} \leq \frac{1+\alpha^{\prime}}{1+\alpha}$. However, since $\frac{q_{k}^{\alpha}}{q_{k}^{\alpha^{\prime}}} \geq 1$ and $\frac{1+\alpha^{\prime}}{1+\alpha}<1$ this gives a contradiction.

According to the mean-value theorem there exists a $q \in\left(q_{k}^{\alpha}, q_{k}^{\alpha^{\prime}}\right)$ such that

$$
C^{\prime}(q)=\frac{C\left(q_{k}^{\alpha^{\prime}}\right)-C\left(q_{k}^{\alpha}\right)}{q_{k}^{\alpha^{\prime}}-q_{k}^{\alpha}},
$$

since the cost function is continuous. Furthermore, it holds that

$$
\begin{aligned}
& C^{\prime}(q)<\max \left\{C^{\prime}\left(q_{k}^{\alpha}\right), C^{\prime}\left(q_{k}^{\alpha^{\prime}}\right)\right\} \\
& \quad \leq P\left((n-k) q_{k}^{\alpha}+k q_{k}^{\alpha^{\prime}}\right) \\
& \Longleftrightarrow P\left((n-k) q_{k}^{\alpha}+k q_{k}^{\alpha^{\prime}}\right) q_{k}^{\alpha^{\prime}}-C\left(q_{k}^{\alpha^{\prime}}\right)>P\left((n-k) q_{k}^{\alpha}+k q_{k}^{\alpha^{\prime}}\right) q_{k}^{\alpha}-C\left(q_{k}^{\alpha}\right),
\end{aligned}
$$

which proves the lemma.

\section{Proof of Lemma 2}

Given a monomorphic state, the pure conjecture dynamics remains in the same monomorphic state with probability one. So $\mathcal{A} \supset\{\{(\alpha, \ldots, \alpha)\} \mid \alpha \in \Lambda\}$. Conversely, let $\alpha \in \Lambda^{n} \backslash \mathcal{A}$. With positive probability all firms may adjust their conjecture and with positive probability all choose the same conjecture, leading to a monomorphic state. Hence,

$$
\mathcal{A} \subset\{\{(\alpha, \ldots, \alpha)\} \mid \alpha \in \Lambda\},
$$

which proves the lemma. 


\section{References}

Alchian, A.A. (1950). Uncertainty, Evolution, and Economic Theory. Journal of Political Economy, 58, 211-221.

Alós-Ferrer, C. , A.B. Ania, and F. Vega-Redondo (1999). An Evolutionary Model of Market Structure. In P.J.J. Herings, G. van der Laan, and A.J.J. Talman (Eds.), The Theory of Markets, pp. 139-163. North-Holland, Amsterdam, The Netherlands.

Ando, A. and F.M. Fisher (1963). Near-Decomposability, Partition and Aggregation, and the Relevance of Stability Discussions. International Economic Review, 4, $53-67$.

Axelrod, R. (1984). The Evolution of Cooperation. Basic Books, New York, NY.

Bauer, F.L. , E. Deutsch, and J. Stoer (1969). Abschätzungen für die Eigenwerte positiver linearer Operatoren. Linear Algebra and Applications, 2, 275-301.

Bowley, A. (1924). The Mathematical Groundwork of the Economics. Oxford University Press, Oxford, UK.

Courtois, P.J. (1977). Decomposability, Queueing and Computer System Applications. ACM monograph series. Academic Press, New York, NY.

Foster, D.P. and H.P. Young (1990). Stochastic Evolutionary Game Dynamics. Theoretical Population Biology, 38, 219-232.

Freidlin, M.I. and A.D. Wentzell (1984). Random Perturbations of Dynamical Systems. Springer-Verlag, Berlin, Germany.

Friedman, J.W. (1967). An Experimental Study of Cooperative Duopoly. Econometrica, 35, 379-397.

Friedman, J.W. and C. Mezzetti (2002). Bounded Rationality, Dynamic Oligopoly, and Conjectural Variations. Journal of Economic Behavior \& Organization, 49, 287-306.

Güth, W. and M. Yaari (1992). An Evolutionary Approach to Explain Reciprocal Behavior in a Simple Strategic Game. In U. Witt (Ed.), Explaining Process and Change - Approaches to Evolutionary Economics. The University of Michigan Press, Ann Arbor, Mich.

Kaarbøe, O.M. and A.F. Tieman (1999). Equilibrium Selection in Supermodular Games with Simultaneous Play. Mimeo, Dept. of Economics, University of Bergen, Bergen, Norway. 
Kalai, E. and W. Stanford (1985). Conjectural Variations in Accelerated Cournot Games. International Journal of Industrial Organization, 3, 133-152.

Kandori, M. , G.J. Mailath, and R. Rob (1993). Learning, Mutation, and Long Run Equilibria in Games. Econometrica, 61, 29-56.

Lancaster, P. and M. Tismentetsky (1985). The Theory of Matrices. Academic Press, Orlando, Fla.

Milgrom, P. and J. Roberts (1991). Adaptive and Sophisticated Learning in Normal Form Games. Games and Economic Behavior, 3, 82-100.

Offerman, T. , J. Potters, and J. Sonnemans (2002). Imitation and Belief Learning in an Oligopoly Experiment. Review of Economic Studies, 69, 973-997.

Schenk-Hoppé, K.R. (2000). The Evolution of Walrasian Behavior in Oligopolies. Journal of Mathematical Economics, 33, 35-55.

Schipper, B.C. (2003). Imitators and Optimizers in Cournot Oligopoly. Mimeo, University of Bonn, Bonn, Germany.

Simon, H.A. (1957). Models of Man. Wiley, New York, NY.

Simon, H.A. and A. Ando (1961). Aggregation of Variables in Dynamic Systems. Econometrica, 29, 111-138.

Vega-Redondo, F. (1997). The Evolution of Walrasian Behavior. Econometrica, 65, 375-384.

Vives, X. (1999). Oligopoly Pricing, Old Ideas and New Tools. MIT-press, Cambridge, Mass.

Young, H.P. (1993). The Evolution of Conventions. Econometrica, 61, 57-84. 